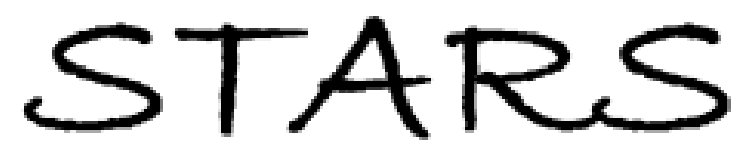

University of Central Florida

STARS

$1-1-2009$

\title{
Nonlinear optical dynamics in nonideal gases of interacting colloidal nanoparticles
}

\author{
R. El-Ganainy \\ University of Central Florida \\ D. N. Christodoulides \\ University of Central Florida \\ E. M. Wright \\ W. M. Lee \\ K. Dholakia
}

Find similar works at: https://stars.library.ucf.edu/facultybib2000

University of Central Florida Libraries http://library.ucf.edu

This Article is brought to you for free and open access by the Faculty Bibliography at STARS. It has been accepted for inclusion in Faculty Bibliography 2000s by an authorized administrator of STARS. For more information, please contactSTARS@ucf.edu.

\section{Recommended Citation}

El-Ganainy, R.; Christodoulides, D. N.; Wright, E. M.; Lee, W. M.; and Dholakia, K., "Nonlinear optical dynamics in nonideal gases of interacting colloidal nanoparticles" (2009). Faculty Bibliography 2000s. 1515.

https://stars.library.ucf.edu/facultybib2000/1515

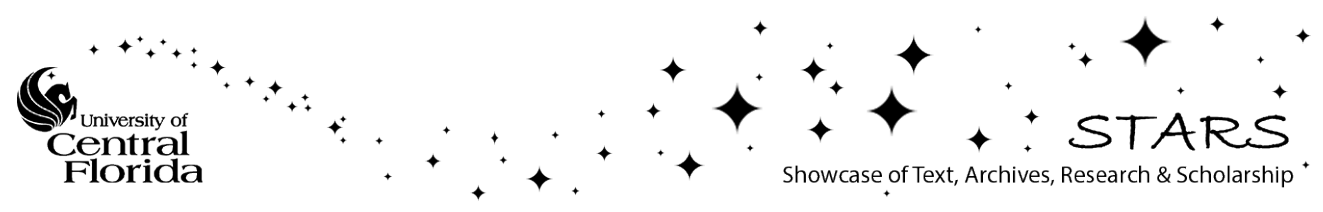




\title{
Nonlinear optical dynamics in nonideal gases of interacting colloidal nanoparticles
}

\author{
R. El-Ganainy and D. N. Christodoulides \\ CREOL/College of Optics \& Photonics, University of Central Florida, Orlando, Florida 32816, USA \\ E. M. Wright \\ College of Optical Sciences, University of Arizona, Tucson, Arizona 85721, USA \\ W. M. Lee and K. Dholakia \\ SUPA, School of Physics and Astronomy, University of St. Andrews, St. Andrews, Fife KY16 9SS, United Kingdom
}

(Received 26 May 2009; published 3 November 2009)

\begin{abstract}
We show that many-body effects in stabilized nanocolloidal suspensions can have a profound effect on their optical nonlinearity. By considering the screened Coulomb repulsions between nanoparticles, we find that the nonlinear optical behavior of these colloids can range from polynomial to exponential depending on their composition and chemistry. The dynamics and stability properties of optical beams propagating in such nonideal gas environments of interacting colloidal particles are investigated. Our analysis provides a theoretical foundation for understanding the recently observed super-Kerr nonlinear optical response of such systems.
\end{abstract}

DOI: 10.1103/PhysRevA.80.053805

PACS number(s): 42.50.Wk, 42.65.Jx, 42.65.Sf, 42.65.Tg

\section{INTRODUCTION}

Light-matter interactions via radiation forces play nowadays a crucial and central role in several areas of physics, chemistry, and biology [1]. One such example is the rich interdisciplinary field of optical traps or tweezers first pioneered by Ashkin et al. [2]. In these early works, the optical self-focusing and four-wave mixing response of colloidal artificial nonlinear systems were also explored in a series of experiments [3-6]. In such settings, the optical nonlinearity is a direct outcome of the electromagnetic gradient force and can be relatively high depending on the size and index contrast of the nanoparticles involved [7,8]. In general, when an optical beam propagates through a colloidal system, the optical gradient force will attract (or repel) the nanoparticles toward (or away from) local intensity maxima. This process, in conjunction with that of Brownian motion, always raises the average refractive index at the beam center $[5,6]$, as shown schematically in Fig. 1. Interest in this area was lately rekindled in a number of experimental [9-11] and theoretical [12] investigations. Interestingly, in most studies, the optical nonlinearity of such nanocolloidal suspensions was a priori taken to be of the Kerr type. Yet, as independently indicated by two research groups $[13,14]$, this simplistic Kerr assumption tends to overlook the underlying Boltzmann distribution and can only be justified when the optical beam intensity is well below a threshold level set by the thermal energy. In fact, if one adopts the Boltzmann statistics, it directly follows that the nonlinearity should instead vary exponentially with intensity $[13,14]$. The exponential facet of this nonlinearity was also suggested in earlier studies using either thermodynamical arguments [5] or by invoking Chandrasekhar's equation [15]. Determining the exact character of this electrostrictive nonlinear mechanism is of importance since it has direct implications on optical beam dynamics in such nanosuspension systems. While the Kerr nonlinear model ignores on an ad hoc basis Boltzmann statistics, the exponential model on the other hand tends to overestimate the severity of the catastrophic beam collapse in both 1D and 2D arrangements. A
Carnahan-Starling model allowing for hard-sphere particleparticle interactions was recently proposed in order to overcome some of these difficulties $[16,17]$. Nonetheless, nonlinearity saturation effects resulting from this latter type of interactions come into play at exceedingly high filling factors, in contradiction with recent experimental observations [18]. In fact, careful Z-scan-like measurements of this nonlinearity point toward a super-Kerr response (monotonically exceeding Kerr) for volume filling factors as low as $0.1 \%-1 \%$ [18]. This leads to several fundamental questions concerning the actual mechanisms behind this process. If indeed the colloidal nonlinear optical response in this range is super-Kerr, then how can this be explained without contradicting Boltzmann thermodynamics?

In this paper, we investigate the nature of the optical nonlinearity in stabilized colloidal systems and provide answers for the above-posed questions. Starting from a "nonideal gas" equation of state and by taking into account the screened Coulomb interactions among suspended nanoparticles, we show that the nonlinear optical behavior of these

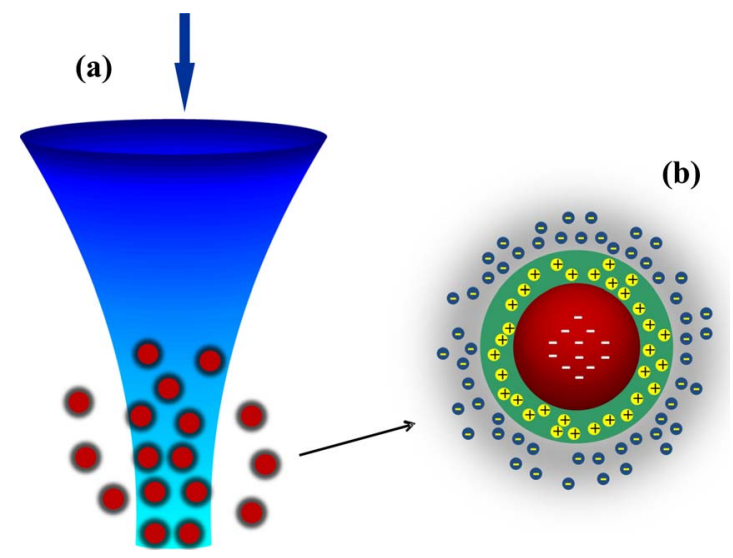

FIG. 1. (Color online) (a) Self-focusing of an optical beam in a colloidal nanosuspension. (b) A charged nanosphere screened by a Gouy-Chapman double layer. 
colloids can range anywhere from exponential to polynomial depending on their filling factor, composition, and chemistry. The thermodynamics of this problem indicate that while the exponential optical nonlinearity is always present, it can be modified by many-body interactions described by Mayer cluster expansions [19]. This in turn has a profound effect on optical beam dynamics. The stability of optical beams in systems with positive polarizabilities is considered in both $1 \mathrm{D}$ and 2D configurations. Our analysis not only provides a foundation for understanding recent experiments but may also open up new opportunities in colloidal science and biophotonics.

\section{NONIDEAL “GAS” EQUATION OF STATE}

Before we proceed, we note that nanocolloids are typically stabilized against coagulation through repulsive electrostatic or entropic forces [20]. Even though here we will focus our attention on electrostatically stabilized colloidal systems, we would like to stress that our analysis is general enough to account for other forms of particle interactions. In general, the repulsive Coulombic force between charged nanoparticles is partly shielded due to the presence of ions in the electrolyte solution. Within the context of Derjaguin, Landau, Verwey, and Overbeek (DLVO) theory - an extension of the Gouy-Chapman double-layer formalism [Fig. 1(b)] - the screened electrostatic interaction between two identical suspended nanoparticles is described by [20]

$$
U(r)=\frac{e^{2} Q^{2}}{4 \pi \varepsilon_{o} \varepsilon_{r}}\left(\frac{e^{\kappa a}}{1+\kappa a}\right)^{2} \frac{e^{-\kappa r}}{r}+W(r),
$$

where $e$ is the magnitude of the electron charge, $Q$ is the number of charges per sphere, $\varepsilon_{0}$ is the free space permittivity, and $\varepsilon_{r}$ is the liquid relative permittivity. In Eq. (1), $a$ is the particle radius, $r$ is the center to center distance between any two spheres, and $W(r)$ represents the van der Waals attractive potential. The Debye-Hückel screening length $l_{D}$ $=1 / \kappa$ associated with the electrolyte solution is given by $\kappa^{2}=\left(e^{2} / \varepsilon_{0} \varepsilon_{r} k_{B} T\right) \sum_{i=1}^{M} z_{i}^{2} n_{i \infty}$. Here, $k_{B}$ is Boltzmann constant, $T$ is the absolute temperature, $z_{i}$ is the ionic valency, and $n_{i \infty}$ is the ionic number concentration at the neutral state. In this work, we consider only binary electrolytes with $M=2$. Note that in aqueous solutions, $n_{i \infty}$ is a direct function of the $\mathrm{pH}$ value and as such is an additional degree of freedom in controlling the optical nonlinear response of the system. For all practical purposes, this interaction energy can be treated as a perturbation on an otherwise "ideal" gas. Using the total Hamiltonian $H=\sum_{i=1}^{N}\left(\vec{p}_{i} \cdot \vec{p}_{i}\right) /\left(2 m_{p}\right)+\sum_{i<j} U\left(\vec{r}_{i}-\vec{r}_{j}\right)$ in the grand canonical partition function of the system $\Xi$ and by considering the thermodynamic limit where $p_{o} V=k_{B} T \ln (\Xi)$, one obtains the equation of state of such nonideal gas of colloidal particles [20,21]. In the above discussion, $U\left(\vec{r}_{i}\right.$ $-\vec{r}_{j}$ ) is the interaction potential between any two particles, $p_{o}$ is the "osmotic" pressure, and $V$ is the total volume. Under these conditions, the system can be effectively described through a virial expansion [19], i.e.,

$$
\frac{p_{o}}{k_{B} T}=\rho+B_{2}(T) \rho^{2}+B_{3}(T) \rho^{3}+\cdots .
$$

In Eq. (2), $\rho$ is the particle density and $B_{2}$ and $B_{3}$ are the second and third virial coefficients, respectively. The second virial coefficient results from two-body interactions and is given by

$$
B_{2}(T)=-2 \pi \int_{0}^{\infty}\left(e^{-U(r) / k_{B} T}-1\right) r^{2} d r .
$$

On the other hand, the third virial coefficient $B_{3}(T)$ arises from three-body effects and can be written as

$$
B_{3}(T)=-1 /(3 V) \int f_{u}\left(\vec{r}_{1}-\vec{r}_{2}\right) f_{u}\left(\vec{r}_{1}-\vec{r}_{3}\right) f_{u}\left(\vec{r}_{2}-\vec{r}_{3}\right) d \vec{r}_{1} d \vec{r}_{2} d \vec{r}_{3},
$$

where $f_{u}(r)=\exp \left[-U(r) / k_{B} T\right]-1$. By adopting center-ofmass coordinates, we find that $B_{3}(T)=-1 / 3 \int f_{u}\left(\vec{r}_{12}\right) f_{u}\left(\vec{r}_{13}\right)$ $f_{u}\left(\vec{r}_{12}-\vec{r}_{13}\right) d \vec{r}_{12} d \vec{r}_{13}$. This last expression can be further simplified using Katsura's Fourier transformation method [22] (see the Appendix) or by using a transformation of coordinates to two cocentral spherical coordinate systems. Equation (2) represents an equation of state for a nonideal gas of colloidal nanoparticles and can be also written in a more compact form $p_{o} / k_{B} T=\rho Z(\rho)$, where $Z(\rho)=1+B_{2}(T) \rho$ $+B_{3}(T) \rho^{2}+\cdots$ is the compressibility factor [20].

\section{GENERALIZED FICK'S LAW}

We will now derive a generalized form of Fick's law for such an equation of state under equilibrium conditions and in the absence of any external forces. From thermodynamical considerations, the Helmholtz free energy is given by $F$ $=-\left.\int P d V\right|_{N}$, where this expression is calculated at constant number of particles $N$. Using $\rho=N / V$, then for constant $N$, $d V=-\left(N / \rho^{2}\right) d \rho$. By substituting this latter expression back in the Helmholtz free energy, it follows that $F=\int P N / \rho^{2} d \rho$ $=k_{B} T N \int \rho Z(\rho) / \rho^{2} d \rho$ or $F=k_{B} T N \int(Z(\rho) / \rho) d \rho$. The chemical potential of the system can then be obtained using

$$
\mu=\partial F /\left.\partial N\right|_{V}=k_{B} T \partial / \partial N\left\{N \int[Z(\rho) / \rho] d \rho\right\} .
$$

For constant $V, \partial / \partial N=(1 / V) \partial / \partial \rho$. By combining the last two expressions, we find that

$$
\mu=k_{B} T\left\{\int[Z(\rho) / \rho] d \rho+(N / V) \partial / \partial \rho \int[Z(\rho) / \rho] d \rho\right\} .
$$

Noting that $N / V=\rho$ and by using $\partial / \partial \rho \int[Z(\rho) / \rho] d \rho=Z(\rho) / \rho$ in Eq. (6), we arrive at

$$
\mu=k_{B} T\left\{\int[Z(\rho) / \rho] d \rho+Z(\rho)\right\} .
$$

If we now let $G(\rho)=\int Z(\rho) / \rho d \rho$, then the equation for the chemical potential reduces to $\mu=k_{B} T[G(\rho)+Z(\rho)]$. We note that the general form of Fick's law in the absence of any external force is given by 


$$
\vec{J}=-\left(D \rho / k_{B} T\right) \nabla \mu,
$$

where $\vec{J}$ is the particle current density and $D$ is the diffusion coefficient. Substituting back the latter expression for the chemical potential in Eq. (8), one finds that $\vec{J}=-D \rho[\nabla G(\rho)$ $+\nabla Z(\rho)]$. Given that $\nabla G(\rho)=\partial G / \partial \rho \nabla \rho$, it follows that Fick's law takes the form $\vec{J}=-D \rho\{[Z(\rho) / \rho] \nabla \rho+\nabla Z(\rho)\}$ $=-D \rho[Z(\rho) \nabla \rho+\rho \nabla Z(\rho)]$ or

$$
\vec{J}=-D[\nabla(\rho Z(\rho))] .
$$

By superimposing the external optical gradient force acting on a nanoparticle, we finally obtain

$$
\vec{J}=\rho \mu_{b} \frac{\alpha}{4} \nabla I-D \nabla[\rho Z(\rho)] .
$$

In Eq. (10), $\mu_{b}$ is the particle's mobility, $\alpha$ is its electric polarizability, and $I$ is the optical-field intensity. For Rayleigh nanoparticles, the electric polarizability is given by $\alpha$ $=3 V_{p} \varepsilon_{0} n_{b}^{2}\left(\frac{m^{2}-1}{m^{2}+2}\right)$, where $V_{p}=4 \pi a^{3} / 3$ is the particle's volume, $\varepsilon_{0}$ is the free space permittivity, and the dimensionless parameter $m=n_{p} / n_{b}$ represents the ratio of the particle's refractive index $n_{p}$ to that of the host medium $n_{b}[4,5]$.

\section{NONLINEAR OPTICAL RESPONSE AND BEAM PROPAGATION EQUATIONS}

Under equilibrium conditions ( $\vec{J}=0)$, Eq. (10) can be integrated and to third order in the virial expansion we find

$$
\begin{aligned}
\frac{\alpha}{4 k_{B} T}\left(I-I_{0}\right)= & \ln \left(f / f_{0}\right)+\frac{2 B_{2} f_{0}}{V_{p}}\left(\frac{f}{f_{0}}-1\right) \\
& +\frac{3 B_{3} f_{0}^{2}}{2 V_{p}^{2}}\left[\left(\frac{f}{f_{0}}\right)^{2}-1\right] .
\end{aligned}
$$

In Eq. (11), $I_{0}$ is an integration constant and can represent in general a flat background intensity at infinity-here taken to be zero. In addition, $f=V_{p} \rho$ is the local volume filling factor while $f_{0}$ stands for the filling factor at infinity where the beam intensity is assumed to vanish $\left(I_{0}=0\right)$. In deriving Eq. (11), Einstein's relation $D / \mu_{b}=k_{B} T$ was used. Note that in the absence of any particle-particle interactions $\left(B_{2,3}=0\right)$, the nonlinear response of Eq. (11) (e.g., the logarithmic term) reduces to the previously considered ideal-gas Boltzmann distribution [13]. We remark that Eq. (11) is a generic result in which the details of the interparticle interactions are contained in the specific values of $B_{2,3}$. In particular, our conclusions below regarding the appearance of a super-Kerr nonlinear response are founded on Eq. (11) and so are not tied to a specific form of interparticle interaction.

To develop the optical beam evolution equation in such systems, we start from the Helmholtz equation $\nabla^{2} E$ $+k_{0}^{2} n_{e f f}^{2} E=0$, where statistically the effective index of the colloidal medium is given by $n_{\text {eff }}=(1-f) n_{b}+f n_{p}$. By using the slowly varying envelope approximation, i.e., $E(x, y, z)$ $=\Phi(x, y, z) \exp \left(i k_{0} n_{b} z\right)$, one finds that the electric field envelope $\Phi(x, y, z)$ obeys the following evolution equation [13]:
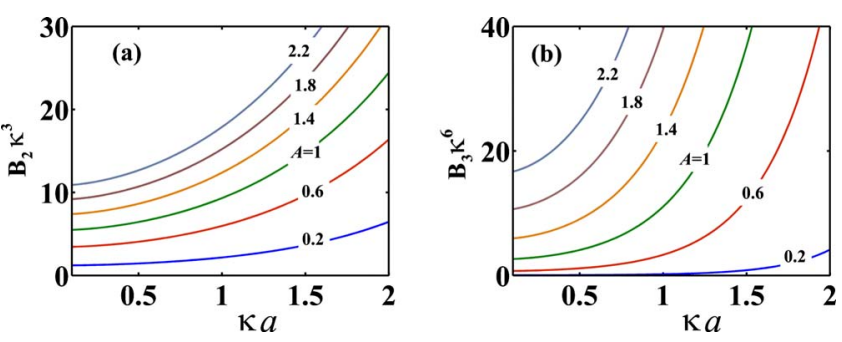

FIG. 2. (Color online) Plots of normalized (a) second and (b) third virial coefficients as a function $\kappa a$ for different values of the constant $A$ (defined above in the text).

$$
i \frac{\partial \Phi}{\partial z}+\frac{1}{2 k_{0} n_{b}} \nabla_{\perp}^{2} \Phi+k_{0}\left(n_{p}-n_{b}\right) f \Phi+\frac{i}{2} \sigma \rho \Phi=0 .
$$

Here, $k_{0}$ is the free space wave number and $\sigma$ is the Rayleigh scattering cross section [13]. By introducing the following normalizations, $\xi=z / z_{0}, z_{0}^{-1}=k_{0}\left|n_{p}-n_{b}\right| f_{0}, X=x / w, Y=y / w$, $w^{2}=z_{0} /\left(2 k_{0} n_{b}\right), \Phi=\left(4 k_{B} T /|\alpha|\right)^{1 / 2} \varphi$, and by keeping in mind that $I=|\Phi|^{2}$, Eqs. (11) and (12) now take the form

$$
\begin{gathered}
i \frac{\partial \varphi}{\partial \xi}+\varphi_{X X}+\varphi_{Y Y}+\operatorname{sgn}(\alpha) \gamma \varphi+i \frac{\sigma}{2 k_{0}\left|n_{p}-n_{b}\right| V_{p}} \gamma \varphi=0, \\
\operatorname{sgn}(\alpha)|\varphi|^{2}=\ln (\gamma)+\frac{2 B_{2} f_{0}}{V_{p}}(\gamma-1)+\frac{3 B_{3} f_{0}^{2}}{2 V_{p}^{2}}\left(\gamma^{2}-1\right) .
\end{gathered}
$$

In Eq. (13), $\gamma=f / f_{0}$ is a filling factor ratio, $\operatorname{sgn}(\alpha)=+1$ when $\alpha>0$ or $n_{p}>n_{b}$, and $\operatorname{sgn}(\alpha)=-1$ for negative polarizabilities, e.g., $n_{p}<n_{b}$. We note that at low densities $\left(f_{0} \rightarrow 0\right)$, the nonlinear response of the system given by Eq. (13b) reduces to the Boltzmann exponential distribution of an ideal gas $[13,14]$. The two coupled Eqs. (13a) and (13b) describe nonlinear optical dynamics in a nonideal gas of interacting nanocolloidal particles. This nonlinear system can be solved numerically in both one-dimensional (1D) and two-dimensional (2D) geometries in order to extract information as to the propagation and stability properties of optical beams in such colloidal suspensions.

\section{RESULTS AND DISCUSSIONS}

To appreciate the effects arising from Coulomb screening, we plot the first two normalized virial coefficients for colloidal systems typically encountered in experiments [Figs. 2(a) and 2(b)]. This is done as function of the ratio between the particle radius and Debye screening length $\kappa a=\left(a / l_{D}\right)$ for various values of the dimensionless interaction potential strength $A=Q^{2}\left(l_{B} / l_{D}\right)$, where $l_{B}=e^{2} /\left(4 \pi \varepsilon_{0} \varepsilon_{r} k_{B} T\right)$ is the socalled Bjerrum length. For simplicity, we have also ignored the van der Waals component in the interaction potential and essentially we employed a linearized Gouy-Chapman double-layer theory or a Debye-Hückel model (DH). As these figures indicate, for a given ratio $\left(a / l_{D}\right)$, the normalized virial coefficients increase as $A$ increases. This should have 

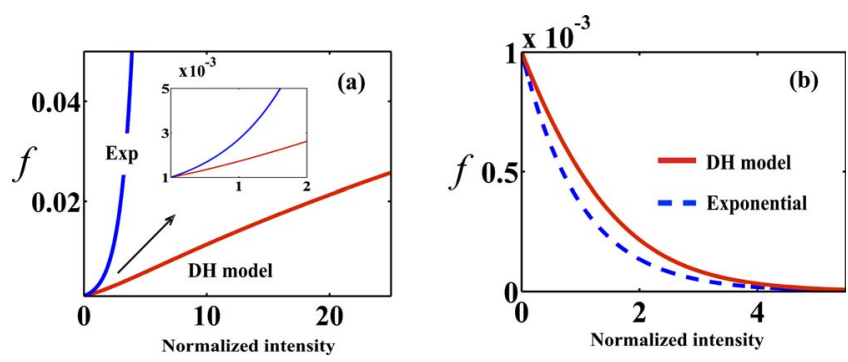

FIG. 3. (Color online) Volume filling factor or nonlinearity vs normalized intensity as obtained from the Debye-Hückel theory (DH) and the Boltzmann exponential model (a) positive polarizabilities and (b) negative polarizabilities. The system parameters are given in the text. Inset in (a) provides an expanded view at low filling factors.

been anticipated given that the interaction strength is directly proportional to $A$. For comparison purposes, we plot both the nonlinear behavior calculated from $\mathrm{DH}$ theory and that obtained from the ideal Boltzmann model for a specific set of parameters. In this example, we assumed an aqueous solution of polystyrene nanoparticles $(a=50 \mathrm{~nm})$ at a $\mathrm{pH}=6$ and a filling factor $f_{0}=10^{-3}$. The average charge per particle was taken here to be $Q=20$. Under these conditions, $B_{2} / V_{p}$ $\approx 300$ and $B_{3} / V_{p}^{2} \approx 7 \times 10^{3}$. For this case, Fig. 3(a) depicts the nonlinear response of this nonideal gas of interacting particles as a function of the normalized intensity $\left(|\alpha| / 4 k_{B} T\right) I$, as obtained from Eq. (11). As evidenced from Eq. (12), the nonlinear index change is always proportional to the filling factor $f$. As Fig. 3(a) indicates, in this range of parameters, the DH curve quickly deviates from the Boltzmann distribution even at very low filling factors, slightly exceeding $f_{0}$-as shown in the inset of Fig. 3(a). This behavior is in contrast to that expected from the Carnahan-Starling hard-sphere model where significant deviations from the exponential model only occur at much higher packing factors-exceeding 5\% [16]. In fact, the competition between the Boltzmann distribution and repulsive interactions leads to an optical response that can range anywhere from exponential to polynomial depending on their filling density, composition, and chemistry. This result explains the artificial Kerr response observed in Refs. [3-5]. In these experiments, the interparticle repulsion acted in such a way so as to soften the exponential nonlinearity and for strong interactions and/or high enough particle concentrations the nonlinear response curve becomes quasilinear, thus giving rise to dominant Kerr effects. It is worth noting that while in [3-5] the artificial Kerr nonlinearity was assumed to be a priori valid, here the same model can emerge naturally from our analysis depending on the strength of interaction between nanocolloidal spheres. An experiment was recently designed to further differentiate between the artificial Kerr regime, the exponential model, and the nonlinear response based on DH interactions [18]. Good agreement between measurements and simulations was obtained when many-body effects were taken into account [18]. More specifically, Lee et al. [18] compared experimental observations to simulations based on the artificial Kerr nonlinearity component [3-5], the exponential nonlinearity, and the DH nonlinear response. In all
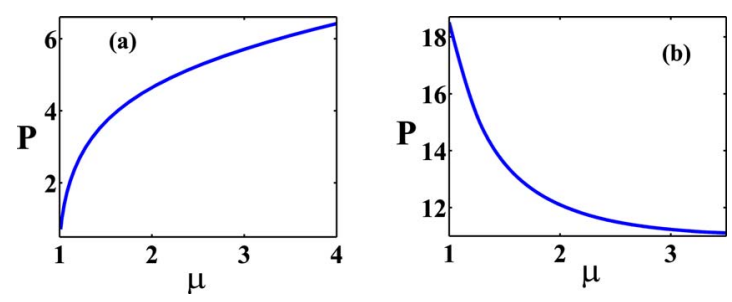

FIG. 4. (Color online) Power-eigenvalue diagrams for (a) onedimensional stripe solitons (b) 2D solitons. The parameters used are identical to those of Fig. 3.

occasions, the DH model was found to be a better fit to the experimental data. Interestingly, even though at high filling densities the exponential distribution can be overshadowed by the higher virial terms, the Kerr coefficient (to lowest order in intensity $I$ ) is largely affected by the Boltzmann term, e.g., $\quad \Delta n(I)=\left(n_{p}-n_{b}\right) f_{0}\left[1+\left(2 B_{2} f_{0} / V_{p}\right)+\cdots\right]^{-1}$ $\left(\alpha I / 4 k_{B} T\right)$. On the other hand, however, for negative polarizabilities, the exponential term dominates the saturable selffocusing optical nonlinearity of this colloidal system, as clearly shown in Fig. 3(b). In this regime, the deviation between the Boltzmann and DH curves is small since the nanoparticles are in this case expelled away from the center of the beam, thus reducing many-particle interaction effects.

We next investigate how many-body interactions may affect the nonlinear dynamics of optical beams propagating in such colloidal systems. A feature that is directly related to the nonlinear response itself is beam stability. To explore optical beam stability in these media, we first consider self-trapped solutions or optical solitons of the form $\varphi=g(X, Y) \exp (i \mu \xi)$ and plot their power-eigenvalue diagrams where the normalized power is given by $P=\int|g|^{2} d X d Y$. Figures 4(a) and 4(b) depict this behavior for both $1 \mathrm{D}$ and $2 \mathrm{D}$ configurations, respectively_obtained for the same parameters used for Fig. 3. From the Vakhitov-Kolokolov criterion [21], one can directly infer that for these specific parameters, onedimensional optical self-trapped channels are stable while their 2D counterparts are unstable. This behavior is consistent with the super-Kerr character of the nonlinear optical response mentioned above. Clearly, this stability behavior can be greatly altered depending on the parameters of the colloidal system itself. For example, 1D solitons can be destabilized if the strength of the interaction is reduced. This can be accomplished either by reducing the initial filling factor (dilute gas) or by altering the chemistry of the solution, thus enhancing screening effects. In that case, the nonlinear response will approach the Boltzmann distribution, rendering even 1D stripe solitons unstable. On the other hand, for 2D self-trapped beams, the presence of interparticle interactions tends to slow down the self-focusing collapse that would have been otherwise severe in a purely exponential model. We note that for negative polarizabilities, the self-focusing nonlinearity is saturable and hence all soliton solutions are stable [13]. The signature of many-body effects on beam propagation can be used to optically extract valuable information concerning the nature of the interaction potential by estimating the virial coefficients. For example, Fig. 5 shows propagation of a 2D optical beam in this same system (corresponding to Fig. 3) at a wavelength of $\lambda=0.532 \mu \mathrm{m}$. Fig- 

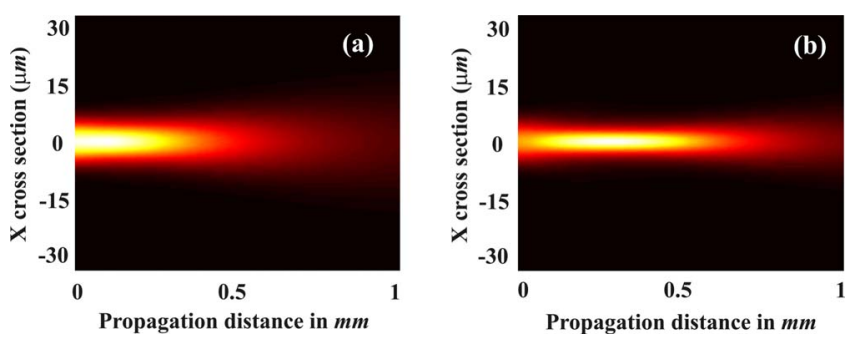

FIG. 5. (Color online) Propagation dynamics of a $7 \mu \mathrm{m}$ FWHM Gaussian beam at a power level of $1 \mathrm{~W}$ (a) for the same system parameters used in Fig. 3 (b) when the charge per particle is reduced to $Q=10$.

ure 5(a) depicts the dynamics of a Gaussian beam of full width at half maximum $(\mathrm{FWHM})=7 \mu \mathrm{m}$ at a power level of $1 \mathrm{~W}$ over a distance of $1 \mathrm{~mm}$ of propagation (3.5 diffraction lengths) when nonlinear Rayleigh losses are taken into account. In this environment, the beam undergoes expansion since the power is not enough to cause self-trapping. Figure 5 (b) on the other hand depicts the intensity evolution of this beam in this same system when the charge per particle is reduced to one half $(Q=10)$. In this case, the optical wave is initially compressed because of reduced interparticle interactions and forms a self-trapped state before nonlinear losses take over. Note that in the absence of nonlinear Rayleigh losses, the beam would have collapsed after a small propagation distance because of the supercritical nature of the optical nonlinearity in 2D. Thus, arrest of supercritical collapse due to nonlinear losses is a key ingredient in understanding how the nominally unstable 2D self-trapped solutions in Fig. 4(b) can nonetheless play a key role in the nonlinear dynamics of optical beams propagating in colloidal suspensions.

\section{CONCLUSIONS}

In conclusion, we have examined the effect of many-body interactions on the optical nonlinearity of nanosuspensions. By considering the screened Coulomb repulsions between nanoparticles, we found that the nonlinear optical behavior of these colloids can range from polynomial to exponential depending on their composition and chemistry. The dynamical behavior of optical beams propagating in such nonideal gas environments of interacting particles was considered and characterized. Furthermore, Eq. (11) is key and shows that inclusion of a number of repulsive interactions provides a theoretical starting point for understanding the super-Kerr nonlinear response observed in recent experiments. Before closing, we would like to mention that other peripheral issues associated with such colloidal systems may merit fur- ther investigation. These include effects arising from the granular distribution of nanoparticles (that could further soften the nonlinear response), rheological corrections, nonlocal thermal effects, as well as nonconservative forces resulting from radiation pressure.

\section{APPENDIX: CALCULATION OF $B_{3}$}

Here we give a brief description of Katsura's Fourier transform method [22] that was used for the calculations of the third virial coefficient $B_{3}(T)$. In order to do so, we start from the expression for $B_{3}(T)$, i.e., $B_{3}(T)$ $=-1 / 3 \int f_{u}\left(\vec{r}_{12}\right) f_{u}\left(\vec{r}_{13}\right) f_{u}\left(\vec{r}_{12}-\vec{r}_{13}\right) d \vec{r}_{12} d \vec{r}_{13}$. If the potential energy between any two particles depends only on their relative positions (as it is in our case) and by using the substitution $\vec{\zeta}_{i}=\vec{r}_{i}-\vec{r}_{1}$, we obtain

$$
B_{3}(T)=-1 / 3 \int f_{u}\left(\left|\vec{\zeta}_{2}\right|\right) f_{u}\left(\left|\vec{\zeta}_{3}\right|\right) f_{u}\left(\left|\vec{\zeta}_{3}-\vec{\zeta}_{2}\right|\right) d \vec{\zeta}_{2} \vec{\zeta}_{3} .
$$

The Fourier transform of $f_{u}(|\vec{\zeta}|)$ can be written as $\tau\left(\vec{k}_{\zeta}\right)$ $=(2 \pi)^{-3 / 2} \int f_{u}(|\vec{\zeta}|) \exp \left(-i \vec{k}_{\zeta} \cdot \vec{\zeta}\right) d \vec{\zeta}$, where $\vec{k}_{\zeta}$ is a conjugate vector in the Fourier space. If we denote $\zeta=|\vec{\zeta}|$ and $k_{\zeta}=\left|\vec{k}_{\zeta}\right|$, then one can write the above expression in the spherical coordinate system $(\zeta, \theta, \phi)$ as

$$
\begin{aligned}
\tau\left(k_{\zeta}\right)= & (2 \pi)^{-3 / 2} \int_{0}^{\infty} \int_{0}^{\pi} \int_{0}^{2 \pi} f_{u}(\zeta) \\
& \times \exp \left[-i k_{\zeta} \zeta \cos (\theta)\right] \zeta^{2} \sin (\theta) d \zeta d \theta d \phi,
\end{aligned}
$$

where in the last equation, $\vec{k}_{\zeta}$ were chosen to coincide with the $\vec{z}$ axis of the spherical coordinate system $(\zeta, \theta, \phi)$. This choice is allowed since the function $f_{u}(|\vec{\zeta}|)$ depends only on the magnitude of $\vec{\zeta}$. The last integral can be further simplified to obtain

$$
\tau(\zeta)=\sqrt{\frac{2}{\pi}} \int_{0}^{\infty} \zeta f_{u}(\zeta) \frac{\sin \left(k_{\zeta} \zeta\right)}{k_{\zeta}} d \zeta .
$$

Similar expression can also be derived for the inverse transform. Substituting in Eq. (A1) and after some mathematical manipulations, it can be shown that

$$
B_{3}=\frac{-(2 \pi)^{3 / 2}}{3} \int_{0}^{\infty} 4 \pi k_{\zeta}^{2} \tau^{3}\left(k_{\zeta}\right) d k_{\zeta} .
$$

Equations (A3) and (A4) can then be used together to obtain numerical values of the third virial coefficient $B_{3}$. Note that by using this technique, the calculations are reduced to only two-dimensional integral [Eqs. (A3) and (A4)] instead of the six dimensional of Eq. (A1).
[1] G. G. Hammes, Thermodynamics and Kinetics for the Biological Sciences (John Wiley and Sons, New York, 2000).

[2] A. Ashkin, J. M. Dziedzic, J. E. Bjorkholm, and S. Chu, Opt. Lett. 11, 288 (1986).
[3] A. Ashkin, J. M. Dziedzic, and P. W. Smith, Opt. Lett. 7, 276 (1982).

[4] P. W. Smith, A. Ashkin, and W. J. Tomlinson, Opt. Lett. 6, 284 (1981). 
[5] P. W. Smith, P. J. Maloney, and A. Ashkin, Opt. Lett. 7, 347 (1982).

[6] P. W. Smith, A. Ashkin, J. E. Bjorkholm, and D. J. Eilenberger, Opt. Lett. 9, 131 (1984).

[7] J. P. Gordon, Phys. Rev. A 8, 14 (1973).

[8] S. Stenholm, Rev. Mod. Phys. 58, 699 (1986).

[9] V. E. Yashin, S. A. Chizhov, R. L. Sabirov, T. V. Starchikova, N. V. Vysotina, N. N. Rozanov, V. E. Semenov, V. A. Smirnov, and S. V. Fedorov, Opt. Spectrosc. 98, 466 (2005).

[10] P. J. Reece, E. M. Wright, and K. Dholakia, Phys. Rev. Lett. 98, 203902 (2007).

[11] J. Junio, E. Blanton, and H. D. Ou-Yang, Proc. SPIE 6644, 664408 (2007).

[12] C. Conti, G. Ruocco, and S. Trillo, Phys. Rev. Lett. 95, 183902 (2005).

[13] R. El-Ganainy, D. N. Christodoulides, C. Rotschild, and M. Segev, Opt. Express 15, 10207 (2007); R. El-Ganainy, D. N. Christodoulides, Z. Musslimani, C. Rotschild, and M. Segev, Opt. Lett. 32, 3185 (2007).

[14] R. Gordon, J. T. Blakely, and D. Sinton, Phys. Rev. A 75,
055801 (2007).

[15] D. Rogovin and S. O. Sari, Phys. Rev. A 31, 2375 (1985).

[16] M. Matuszewski, W. Krolikowski, and Y. S. Kivshar, Opt. Express 16, 1371 (2008); Phys. Rev. A 79, 023814 (2009).

[17] N. F. Carnahan and K. E. Starling, J. Chem. Phys. 51, 635 (1969).

[18] W. M. Lee, R. El-Ganainy, D. N. Christodoulides, K. Dholakia, and E. M. Wright, Opt. Express 17, 10277 (2009).

[19] J. E. Mayer and M. G. Mayer, Statistical Mechanics (John Wiley and Sons, Inc., New York, 1966).

[20] W. B. Russel, D. A. Savillie, and W. R. Schrowalter, Colloidal Dispersion (Cambridge University Press, New York, 1989); J. H. Masliyah and S. Bhattacharjee, Electrokinetic and Colloid Transport Phenomena (John Wiley and Sons, New York, 2006).

[21] N. G. Vakhitov and A. A. Kolokolov, Izv. Vyssh. Uchebn. Zaved., Radiofiz. 16, 1020 (1973); Radiophys. Quantum Electron. 16, 783 (1973).

[22] S. Katsura, Phys. Rev. 115, 1417 (1959). 